\title{
BMJ Open Integrating the prevention and control of rheumatic heart disease into country health systems: a systematic review protocol
}

\author{
Jessica Abrams, ${ }^{1}$ David Watkins, ${ }^{2,3}$ Leila H Abdullahi, ${ }^{1,4}$ Mark E Engel, ${ }^{3}$ \\ Liesl J Zuhlke 1,5
}

To cite: Abrams J,

Watkins D, Abdullahi LH, et al. Integrating the prevention and control of rheumatic heart disease into country health systems: a systematic review protocol. BMJ Open 2019;9:e028908. doi:10.1136/ bmjopen-2019-028908

- Prepublication history and additional material for this paper are available online. To view these files, please visit the journal online (http://dx.doi. org/10.1136/bmjopen-2019028908).

Received 2 January 2019 Revised 19 April 2019 Accepted 21 May 2019
Check for updates

(c) Author(s) (or their employer(s)) 2019. Re-use permitted under CC BY-NC. No commercial re-use. See rights and permissions. Published by BMJ.

For numbered affiliations see end of article.

Correspondence to Dr Liesl J Zuhlke;

liesl.zuhlke@uct.ac.za, ZUHLKE@TELKOMSA.NET

\section{ABSTRACT}

Introduction Rheumatic heart disease (RHD) is a preventable chronic condition affecting the valves of the heart. RHD prevention and care programmes have historically originated in more developed countries, implemented in a targeted (or vertical) manner and evaluated using non-controlled approaches. Taking a broad view of the integration of RHD activities within the whole system is critical for health planning in low-income regions with a high burden of RHD and less robust health systems. Therefore, we propose to conduct a systematic review to assess RHD programme models in order to gain a better understanding of the extent of integration within relevant health systems.

Methods and analysis A predefined search strategy will be used to search for relevant articles published in English from January 1990 to December 2017. Electronic databases PubMed, Scopus, Web of Science, Africa Wide, CINAHL, Cochrane Central Register of Controlled Trials, Google Scholar and Global Index Medicus will be searched, as well as reference lists of relevant articles published. A standardised data extraction form will be used to obtain information for analysis from the included studies. The quality, reliability and risk of bias of included studies will be assessed using designspecific criteria. Programme integration will be analysed according to stewardship and governance, financing, planning, service delivery, monitoring and evaluation, and demand generation. Programme inputs, outputs and impact will also be described.

Ethics and dissemination No ethical approval is required. Findings will be disseminated in a peer-review journal in accordance with the Preferred Reporting Items for Systematic reviews and Meta-Analyses guidelines. PROSPERO registration number CRD42017076307

\section{INTRODUCTION}

Rheumatic heart disease (RHD) is a preventable chronic cardiovascular condition which affects more than 30 million individuals worldwide and is responsible for about 300000 deaths annually. ${ }^{1}$ These deaths mostly occur among children and young adults living in socioeconomically disadvantaged communities, such as those found in low-income to middle-income countries and among indigenous populations
Strengths and limitations of this study

- To our knowledge, this will be the first systematic review to analyse the integration of prevention and control programmes for rheumatic heat disease (RHD).

- By using multiple complementary conceptual frameworks (health system critical functions, type of services delivered, and results chain) to assess each programme, our study will provide a unique and comprehensive analysis of the landscape of RHD programmatic activities.

- Our analysis will guide the development and evaluation of integrated RHD programmes in low-income and middle-income countries experiencing endemic patterns of RHD.

- We recognise that restricting the search to English may introduce language and publication bias, and that the time period restriction will exclude older studies that may contain important historical information and experience, predominately from high-income countries.

of some high-income countries. ${ }^{1}$ Overcrowded living conditions and limited access to healthcare services are important risk factors for the eventual development of RHD. ${ }^{23}$ The infectious agent, group A $\beta$-haemolytic streptococcus (Strep A), is easily transmitted between people in close contact with an infected individual. ${ }^{4}$ When Strep A infection is left untreated, acute rheumatic fever (ARF) may arise due to an autoimmune response within the body. ${ }^{5}$ Following one or more recurrent episodes of ARF, the heart valves may incur permanent damage known as RHD.

There are multiple opportunities to intervene along the Strep A to RHD pathway and prevent morbidity and mortality; however, progress in implementing interventions has been uneven. Comprehensive RHD control encompasses the whole health system, from primary care to specialised tertiary care and therefore requires 
a more complete, integrated approach to patient management. ${ }^{6}$ Integration is, thus, required in two dimensions: first, along the patient continuum of care (from Strep A to RHD and primary care to tertiary care), and second, with other health system activities (eg, maternal health, child and adolescent health, and control of other non-communicable diseases). Historically, RHD control programmes were often delivered in a relatively targeted, or vertical, fashion and were focused mostly on secondary prevention ${ }^{7}$ or a combination of secondary and primary prevention. ${ }^{8}$ However, the broad consensus among health systems experts is that 'horizontal' or 'diagonal' programmatic approaches are more sustainable, particularly as country health systems become more advanced and begin to tackle chronic non-communicable diseases. ${ }^{9}$ Additionally, much of the RHD programmatic research emerged from developed settings several decades ago and did not use controlled approaches, limiting its applicability to populations in less-resourced settings that have weak health systems. ${ }^{10}{ }^{11}$ As a result, there is little evidence to guide the development and sustained implementation of comprehensive, integrated RHD control programmes in the countries where RHD remains a public health concern.

We propose to conduct a systematic review to assess the literature, synthesising qualitative and qualitative data on programmes addressing RHD prevention and control, and assess these programmes on the basis of their integration in terms of critical health system elements and on the basis of their reported performance (outputs, outcomes and impact). This review will identify the best practices in RHD care and provide technical assistance to countries seeking to effectively and efficiently integrate RHD-related health services into existing health systems.

\section{METHODS AND ANALYSIS}

The protocol is reported in compliance with the Preferred Reporting Items for Systematic Reviews and Meta-Analyses Protocols (PRISMA) guidelines. The completed checklist can be found in online supplementary appendix $1 .^{12}$

\section{Patient and public involvement}

There is no direct patient or public involvement in this study.

\section{Objective}

To assess integrated programmes targeting RHD prevention and control in order to evaluate the effectiveness of the extent and nature of integration within the relevant health systems.

\section{Eligibility criteria of studies for this review \\ Study designs}

A combination of analytical and descriptive studies will be considered for this review. These include randomised controlled trials and controlled clinical trials, quasi-experimental, controlled before-and-after studies, interrupted time series designs, cross-sectional studies. 'Opinion pieces', narrative reviews and letters to the editor will not be included.

\section{Participants and interventions}

We will consider any study that reports on a health programme directed at populations at risk for Strep A infection, rheumatic fever and/or RHD. Importantly, we will focus on the changes in health service delivery brought about by the programme and the downstream impact on intermediate and final health outcomes. Therefore, the unit of analysis for this review will be the programme rather than the individual receiving the intervention. A programme will be defined as a coherent and intentional effort to expand health services to the population. We will use commonly agreed-upon classifications for RHD-related services: primary prevention, secondary prevention and advanced medical or surgical care. For non-experimental studies, we do not expect to record comparators. Where relevant (ie, in experimental and quasi-experimental designs), the comparator will be locations or populations where the RHD programme is not in place.

\section{Primary outcomes}

\section{Programme characteristics}

Programme characteristics will include: the programme start year, location(s), duration, area of emphasis (services delivered: primary, secondary, tertiary) and inputs.

\section{Programme integration}

We will make use of a framework developed by Atun et al to determine the extent of integration into the existing health system and assign scores based on the extent of integration. ${ }^{13}$ Integration will be defined as 'the extent, pattern and rate of adoption and eventual assimilation of health interventions into each of the critical functions of a health system'. ${ }^{13}$ Six critical health system functions are delineated: stewardship and governance, financing, planning, service delivery, monitoring and evaluation, and demand generation. ${ }^{13}$ In a subsequent systematic review, Atun et aldemonstrated how this framework could be used to evaluate the extent of programme integration in each of these dimensions and across different health concerns. ${ }^{14}$ Further details on how integration is assessed for each of the functions is outlined in online supplementary appendix 2.

\section{Secondary outcome}

\section{Programme results}

We will use a 'results chain' framework depicted in figure 1 to identify and describe the effects of various programmes. ${ }^{15}$ Results chains are commonly used tools for evaluating the impact of health programmes. The results chain consists of inputs (such as human and physical resources), which produce outputs (such as the volume and scope of services produced), which lead to outcomes (such as changes in behaviours or physiologic indicators of risk), which lead 


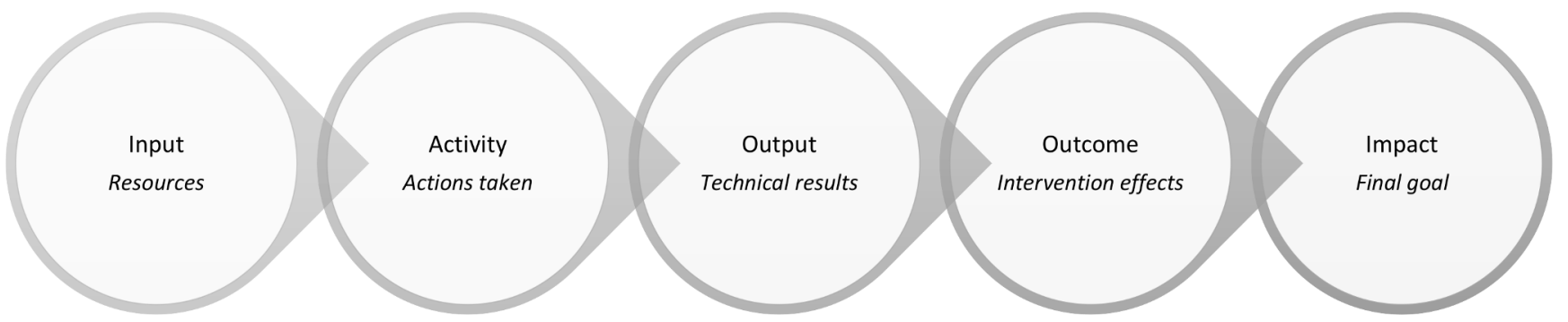

Figure 1 The results chain framework.

to final impacts (such as reductions in disease-specific incidence and mortality rates).

\section{Search strategy}

A comprehensive search strategy has been developed to systematically search PubMed, Cochrane Central Register of Controlled Trials, Scopus, ISI Web of Science, Africa Wide and CINAHL. Google Scholar and Global Index Medicus (which includes Latin America and the Caribbean database LILACS, as well as WHO Library Information System will be searched for grey literature. The search will include Medical Subject Headings and freeterm text items, published in English from 1990 onward. These restrictions have been chosen given that we seek contemporary prevention and treatment programmes with limited time and resources. Table 1 displays the specific search strategy for PubMed that will be adapted for other databases. The reference lists of included papers will be handsearched for relevant studies. Published and unpublished data will be sourced by contacting authors, as well as by screening abstracts from the latest relevant conferences.

\section{Exclusion criteria}

Studies will be excluded should they be published prior to January 1990 or after December 2017, if they do not correspond to the aforementioned study designs, or if they do not display a clear effort to expand health services to people at risk for Strep A, ARF and or RHD. Studies with insufficient information on programme characteristics will be excluded, as well as studies reporting on fewer than four of the six key functions of the health system.

\section{Selection of studies}

The first author (JA) will perform a systematic search for articles using the search strategy. Two reviewer authors (JA and DW) will then independently screen the titles and abstracts of the search results using the predefined inclusion and exclusion criteria (online supplementary appendix 3, sections A and B). Reasons for exclusion will be documented. Any discrepancies will be discussed and where consensus cannot be reached, resolved by a third author. Articles finalised for inclusion will be retrieved and managed with Mendeley reference manager. A flow chart will be presented to summarise the search process and selection of studies for this review. This is in keeping with PRISMA guidelines.

\section{Data extraction and management}

Two reviewer authors will independently extract data using the predesigned data extraction form (online supplementary appendix 3 , sections $\mathrm{A}$ and $\mathrm{E}$ ), which will be piloted beforehand with five studies to ensure its validity. Data extraction discrepancies will be ameliorated through discussion, and where contradictions still remain, a third reviewer will be consulted. In addition to the details pertaining to basic study characteristics (author, study design, duration and location of the study) and information for assessment of risk of bias, we will extract a variety of quantitative and qualitative data related to our primary and secondary outcomes (online supplementary appendix 3, section D). Data will be extracted in accordance with our conceptual frameworks-health system key functions, type of services delivered and results chain. Certain data might not be reported by a study, such as in the case of outcomes or impacts of the results chain; this

Table 1 PubMed search strategy

\section{Search Terms}

1. Pharyngitis[MeSH Terms] OR rheumatic heart disease[MeSH Terms] OR rheumatic fever[MeSH Terms] OR pharyng*[Title/ Abstract] OR "sore throat"[Title/Abstract] OR "group A strep*“[Title/Abstract] OR "rheumatic fever"[Title/Abstract] OR "rheumatic heart disease"[Title/Abstract] OR RHD[Title/Abstract]

2. (preventative health services[MeSH Terms]) OR delivery of health care, integrated([MeSH Terms]) OR healthcare[Title/ Abstract]) OR health care[Title/Abstract]) AND (vertical[Title/Abstract] OR horizontal[Title/Abstract] OR integrated[Title/Abstract] OR coordinat*[Title/Abstract] OR co-ordinat*[Title/Abstract] OR program*[Title/Abstract] OR service*[Title/Abstract]) 
will be noted during the data extraction as unreported. Where study data are unclear, the author of the manuscript will be contacted.

\section{Data synthesis and analysis}

This review will synthesise study data using qualitative and quantitative approaches. We will transform programme characteristics into descriptive statistics, such as the proportion of studies focusing on primary prevention. We will create a series of data display matrices, one for each type of clinical service delivered; namely, primary, secondary or tertiary levels of prevention, or combinations thereof. The matrices will present qualitative and quantitative data along the results chain (columns) for each study (rows) that provide data on the respective programmes.

Where appropriate, we will conduct meta-analyses on the change of effect at different points along the results chain, focusing on outcomes (ie, intermediate clinical outcomes such as the detection of new RHD cases) and impact on population health (ie, disease incidence, disability and mortality rates). Care will be taken to pool results only from programmes that have similar models of care delivery; otherwise, we will provide a narrative review of programme outcomes.

Outcome data of included studies will be expressed as a risk ratio with corresponding $95 \%$ CI for dichotomous data, or mean difference and SD for continuous data. Where outcomes are measured using different scales, the standardised mean difference will be reported. A random effects meta-analysis will be performed according to the Mantel-Haenszel method in the absence of statistical heterogeneity, methodological difference or high risk of bias. Should the included studies be of substantial heterogeneity and where statistical pooling is not possible, results will be presented in a narrative format, including suitable tables and figures.

Heterogeneity will be evaluated by examining population characteristics, approaches to delivery of interventions and differences in definitions or measurement of study outcomes. Heterogeneity of the programme effects across studies will be assessed visually by analysing the forest plot, the $\mathrm{X}^{2}$ test having a $10 \%$ level of significance and using the $\mathrm{I}^{2}$ statistic with cut-offs of $25 \%, 50 \%$ and $75 \%$ representing low, medium and high heterogeneity, respectively.

Our second primary objective, to assess the extent of programme integration into existing health systems, will be measured by assigning a score from 1 to 3 for programmes which are not integrated, partially integrated or fully integrated into the existing health system for each of the six key dimensions described previously. ${ }^{13}$ Thereafter, a composite score with a maximum value of 18 will be determined and compared. If the number of similar studies is sufficient to conduct meta-analyses, we will attempt to identify the influence of programme integration on study outcomes by using either subgroup analysis or random-effects meta-regression with our integrations core included as a covariate. Again if data are limited or too heterogeneous to pool, we will summarise our findings in a narrative format.

\section{Risk of bias and quality appraisal of included studies}

The Critical Appraisal Skills Programme checklist will be used to assess the risk of bias of experimental and observational studies included in this review (online supplementary appendix 3 , section $\mathrm{E}$ ).

Information will be gathered on randomisation sequence generation, allocation concealment, masking of study participants and personnel, completeness of outcome data, selective outcome reporting and any other sources of bias. The risk of bias assessment will be accompanied by a summary of the reasoning behind the decision. Each included study will be labelled as having low, unclear or high risk of bias and will be presented in a figure. Any discrepancies of bias assessment will be resolved through discussion or subsequent consultation with a third author.

In order to minimise publication biases, this study will employ strategies to search for, and include unpublished studies such as those found in grey literature. A funnel plot will be used to assess the risk of publication bias which will be critically examined for asymmetry both visually and through the use of formal tests.

Notably, we anticipate finding a variety of descriptive, non-experimental studies. We will extract data relevant to our primary and secondary outcomes above but not attempt to quantify meta-bias in this subset of studies.

The quality of evidence will be assessed using The Grading of Recommendations Assessment, Development and Evaluation approach. Evidence will be graded as very low, low, moderate or high quality.

\section{Dissemination and anticipated impact}

A previous review was conducted on interventions for neglected tropical diseases, nutrition, immunisation, child health and development, family planning and HIV/AIDS. ${ }^{14}$ Contrary to the popular notion that health programmes are either uniformly vertical (targeted) or horizontal (integrated), it was found that most programmes were partially integrated, and that the extent of integration varied according to each of the six critical health system functions. It was strongly argued that such heterogeneity was desirable given differences in health system design, capacity and priorities. ${ }^{14}$ For some programmes, such as child immunisation, targeted campaigns may actually be more effective, efficient and sustainable. For other programmes, such as those that provide clinical care for diabetes and other complex chronic diseases, episodic community-based activities are unlikely to achieve significant health impact or be financially sustainable and integrated approaches delivered at primary health centres are needed.

Among non-communicable diseases, RHD and its antecedents represent a unique set of considerations that span a wide spectrum of health system activities. Some 
aspects of RHD prevention have much in common with infectious disease control. For example, surveillance and notification policies are needed in order to identify and respond quickly to rheumatic fever outbreaks and ramp up primary prevention activities. Other aspects-such as secondary prevention-have more in common with clinic-based care for hypertension, diabetes and HIV/AIDS. The frequent need for (highly-effective) tertiary surgical and medical care also creates additional complexity. In light of all this, there is unlikely to be a 'one size fits all' approach to RHD prevention and control in low-income and middle-income countries. What is needed, which we believe this systematic review will begin to provide, is evidence regarding efficient models of care and best practice. A robust analysis of the purpose, extent and nature of integration for programmes and services will be of interest to decision makers in resource-constrained settings as well as those in more developed regions wishing to scale up RHD-related activities. We anticipate that this review will raise more questions than propose solutions, however, and view this as a first step in an empirical research agenda that will involve prospective studies in a variety of country contexts.

\section{Author affiliations}

${ }^{1}$ Department of Paediatrics, Red Cross War Memorial Children's Hospital, Cape Town, Western Cape, South Africa

${ }^{2}$ Department of Medicine, University of Washington, Seattle, WA, United States ${ }^{3}$ Department of Medicine, University of Cape Town, Western Cape, South Africa ${ }^{4}$ Research, Evaluation, Analysis, Learning and Monitoring (REALM), Save the Children International, Nairobi, Kenya

${ }^{5}$ Department of Medicine, Groote Schuur Hospital, Cape Town, South Africa

Contributors DW and LJZ conceived the review. JA and DW developed and wrote the first draft. LHA and MEE provided key scientific input and oversight regarding data extraction form development. LHA, MEE and LJZ edited the subsequent versions of the draft. All authors approved the final draft and the revision document. LJZ assisted JA in revising the protocol and takes overall responsibility for the manuscript. DW, LHA, MEE and LJZ supervised the overall development of the protocol.

Funding LJZ and DW receive funding from the Medtronic Foundation through support to RHD Action. JA is the recipient of a scholarship from the National Research Foundation of South Africa (NRFSA). LJZ is also funded by the National Research Foundation of South Africa and The Medical Research Council of South Africa.

Competing interests None declared.

Patient consent for publication Not required.
Ethics approval No formal ethical review is required due to the nature of the systematic review which draws on publicly available data. Findings will be disseminated in a peer-review journal in accordance with the Preferred Reporting Items for Systematic reviews and Meta-Analyses (PRISMA) guidelines.

Provenance and peer review Not commissioned; externally peer reviewed.

Open access This is an open access article distributed in accordance with the Creative Commons Attribution Non Commercial (CC BY-NC 4.0) license, which permits others to distribute, remix, adapt, build upon this work non-commercially, and license their derivative works on different terms, provided the original work is properly cited, appropriate credit is given, any changes made indicated, and the use is non-commercial. See: http://creativecommons.org/licenses/by-nc/4.0/.

\section{REFERENCES}

1. Watkins DA, Johnson CO, Colquhoun SM, et al. Global, Regional, and National Burden of Rheumatic Heart Disease, 1990-2015. N Engl J Med 2017;377:713-22.

2. Jaine R, Baker M, Venugopal K. Acute rheumatic fever associated with household crowding in a developed country. Pediatr Infect Dis $J$ 2011;30:315-9.

3. Riaz BK, Selim S, Karim MN, et al. Risk factors of rheumatic heart disease in Bangladesh: a case-control study. $J$ Health Popul Nutr 2013;31:70-7.

4. Patterson MJ. Streptococcus: In. Baron S, ed. Medical Microbiology, 1996. http://www.ncbi.nlm.nih.gov/pubmed/21413248. (Accessed 22 Jan 2018).

5. Sika-Paotonu D, Beaton A, Raghu A, et al. In: Ferretti J, Stevens D, Fischetti V, eds. Streptococcus pyogenes: Basic Biology to Clinical Manifestations. Oklahoma City (OK: University of Oklahoma Health Sciences Center, 2016:1-57. Updated 3 Apr 2017.

6. Palafox B, Mocumbi AO, Kumar RK, et al. The WHF Roadmap for Reducing CV Morbidity and Mortality Through Prevention and Control of RHD. Glob Heart 2017;12:47-62.

7. Strasser T, Dondog N, El Kholy A, et al. The community control of rheumatic fever and rheumatic heart disease: report of a WHO international cooperative project. Bull World Health Organ 1981;59:285-94.

8. Nordet P, Lopez R, Dueñas A, et al. Prevention and control of rheumatic fever and rheumatic heart disease: the Cuban experience (1986-1996-2002). Cardiovasc J Afr 2008;19:135-40.

9. Knaul FM, Bhadelia A, Atun R, et al. Achieving Effective Universal Health Coverage And Diagonal Approaches To Care For Chronic Illnesses. Health Aff 2015;34:1514-22.

10. RHD Action. RHD Global Status Report 2015-17, 2015.

11. Gordis L. The virtual disappearance of rheumatic fever in the United States: lessons in the rise and fall of disease. T. Duckett Jones memorial lecture. Circulation 1985;72:1155-62.

12. Moher D, Shamseer L, Clarke M, et al. Preferred reporting items for systematic review and meta-analysis protocols (PRISMA-P) 2015 statement. Syst Rev 2015;4:1.

13. Atun R, de Jongh T, Secci F, et al. Integration of targeted health interventions into health systems: a conceptual framework for analysis. Health Policy Plan 2010;25:104-11.

14. Atun $\mathrm{R}$, de Jongh $\mathrm{T}$, Secci $\mathrm{F}$, et al. A systematic review of the evidence on integration of targeted health interventions into health systems. Health Policy Plan 2010;25:1-14.

15. Gertler PJ, Martinez S, Premand P, et al. Impact Evaluation in Practice. Washington DC: The World Bank, 2011. 